\title{
CORRIGENDUM TO "ATOMIC DECOMPOSITION OF HARDY-MORREY SPACES WITH VARIABLE EXPONENTS"
}

\section{Kwok-Pun Ho}

The Education University of Hong Kong, Department of Mathematics and Information Technology 10 Lo Ping Road, Tai Po, Hong Kong, P. R. China; vkpho@eduhk.hk

Abstract. We correct a technical error in [2, Theorem 5.1].

In this note, we correct a technical error appeared in the proof of [2, Theorem 5.1]. At the end of p. 49 of [2], we use the inequalities

$$
\begin{aligned}
c\left|\varphi * g^{j}(x)\right| & \leq\left(\mathcal{M} g^{j}\right)(x) \\
& \leq(\mathcal{M} f)(x) \chi_{\left\{x \in \mathbf{R}^{n}:(\mathcal{M} f)(x) \leq 2^{j}\right\}}(x)+2^{j} \sum_{k \in \mathbf{N}} \frac{l\left(Q_{k}^{j}\right)^{n+d+1}}{\left.l\left(Q_{k}^{j}\right)+\left|x-x_{k}^{j}\right|\right)^{n+d+1}} \\
& \leq C 2^{j}
\end{aligned}
$$

to prove that $g^{j} \rightarrow 0$ in $\mathcal{S}^{\prime}\left(\mathbf{R}^{n}\right)$ as $j \rightarrow-\infty$. This is an error as the last inequality does not necessarily hold.

Most importantly, the result $\lim _{j \rightarrow-\infty} g^{j}=0$ in $\mathcal{S}^{\prime}\left(\mathbf{R}^{n}\right)$ is valid. We now give a proof of the result $\lim _{j \rightarrow-\infty} g^{j}=0$ in $\mathcal{S}^{\prime}\left(\mathbf{R}^{n}\right)$ by using the ideas in [2, p. 50]. The reader is referred to [2] for the notions used in this note.

For any $Q \in \mathbf{B},[2$, Propoisition 5.4] yields

$$
\begin{aligned}
\int_{Q}\left|\left(\mathcal{M} g^{j}\right)(x)\right| d x & \leq C 2^{j} \int_{Q} d x+C 2^{j} \int_{Q} \sum_{k \in \mathbf{N}} \frac{l\left(Q_{k}^{j}\right)^{n+d_{p(\cdot)+1}}}{\left(l\left(Q_{k}^{j}\right)+\left|x-x_{k}^{j}\right|\right)^{n+d_{p(\cdot)}+1}} d x \\
& \leq C 2^{j}|Q|+C 2^{j} \sum_{k \in \mathbf{N}} \int_{\mathbf{R}^{n}} \chi_{Q}(x)\left(\left(\mathrm{M} \chi_{Q_{k}^{j}}\right)(x)\right)^{\left(n+d_{p(\cdot)+1) / n}\right.} d x .
\end{aligned}
$$

By using [1, Chapter II, Theorem 2.12], we obtain

$$
\begin{aligned}
\int_{\mathbf{R}^{n}}\left(\left(\mathrm{M} \chi_{Q_{k}^{j}}\right)(x)\right)^{\left(n+d_{p(\cdot)}+1\right) / n} \chi_{Q}(x) d x & \leq \int_{\mathbf{R}^{n}}\left(\chi_{Q_{k}^{j}}(x)\right)^{\left(n+d_{p(\cdot)}+1\right) / n}\left(\mathrm{M} \chi_{Q}\right)(x) d x \\
& =\int_{\mathbf{R}^{n}} \chi_{Q_{k}^{j}}(x)\left(\mathrm{M} \chi_{Q}\right)(x) d x \\
& =\int_{Q_{k}^{j}}\left(\mathrm{M} \chi_{Q}\right)(x) d x
\end{aligned}
$$

because $\left(n+d_{p(\cdot)}+1\right) / n>1$.

https://doi.org/10.5186/aasfm.2017.4219

2010 Mathematics Subject Classification: Primary 42B20, 42B25, 42B35, 46E30.

Key words: Atomic decomposition, Morrey spaces, Hardy spaces, maximal functions, variable exponent analysis, vector-valued maximal inequalities. 
Therefore, the finite intersection property of $\left\{Q_{k}^{j}\right\}$ yields

$$
\begin{aligned}
\int_{Q}\left|\left(\mathcal{M} g^{j}\right)(x)\right| d x & \leq C 2^{j}|Q|+C 2^{j} \sum_{k \in \mathbf{N}} \int_{Q_{k}^{j}}\left(\mathrm{M} \chi_{Q}\right)(x) d x \\
& \leq C 2^{j}|Q|+C 2^{j} \int_{O^{j}}\left(\mathrm{M} \chi_{Q}\right)(x) d x .
\end{aligned}
$$

Consequently, for any $\varphi \in \mathcal{S}\left(\mathbf{R}^{n}\right)$ and $x \in \mathbf{R}^{n}$, we have

$$
\begin{aligned}
\left|g^{j} * \varphi(x)\right| & \leq C \frac{1}{|B(x, 1)|} \int_{B(x, 1)}\left|M_{1}^{*}\left(g^{j}, \varphi\right)(y)\right| d y \\
& \leq C \int_{B(x, 1)}\left|\left(\mathcal{M} g^{j}\right)(y)\right| d y \\
& \leq C 2^{j}|B(x, 1)|+C 2^{j} \int_{O^{j}}\left(\mathrm{M} \chi_{B(x, 1)}\right)(y) d x
\end{aligned}
$$

for some $C>0$.

Thus, it suffices to show that $2^{j} \int_{O^{j}}\left(\mathrm{M} \chi_{B(x, 1)}\right)(y) d x \rightarrow 0$ as $j \rightarrow-\infty$.

Let $0<r<\min \left(1, m_{p(\cdot)}\right)$ and $B^{k}=B\left(x, 2^{k}\right) \backslash B\left(x, 2^{k-1}\right)$ when $k \geq 1$ and $B^{0}=$ $B(x, 1)$. We find that

$$
\begin{aligned}
& \int_{O^{j}}\left(\mathrm{M} \chi_{B(x, 1)}\right)(y) d x \leq \int_{O^{j}}(1+|x-y|)^{-n} d y \leq C \sum_{k=0}^{\infty} 2^{-k n} \int_{O^{j}} \chi_{B^{k}}(y) d y \\
& \leq C \sum_{k=0}^{\infty} \frac{1}{\left|B\left(x, 2^{k}\right)\right|}\left\|\chi_{O^{j} \cap B\left(x, 2^{k}\right)}\right\|_{L^{p(\cdot) / r}\left(\mathbf{R}^{n}\right)}\left\|\chi_{B\left(x, 2^{k}\right)}\right\|_{L^{(p(\cdot) / r)^{\prime}}\left(\mathbf{R}^{n}\right)} \\
& \leq C \sum_{k=0}^{\infty} \frac{u\left(x, 2^{k}\right)^{r}}{\left\|\chi_{B\left(x, 2^{k}\right)}\right\|_{L^{p(\cdot) / r}\left(\mathbf{R}^{n}\right)}}\left\|\chi_{O^{j}}\right\|_{\mathcal{M}_{p(\cdot), u}}^{r} .
\end{aligned}
$$

As $u^{r} \in \mathcal{W}_{h_{p(\cdot) / r}},[2$, Lemma 3.3] yields

$$
2^{j} \int_{O^{j}}\left(\mathrm{M} \chi_{Q(x, 1)}\right)(y) d x \leq 2^{j} C\left\|\chi_{O^{j}}\right\|_{\mathcal{M}_{p(\cdot), u}}^{r}
$$

for some $C>0$ independent of $j \in \mathbf{Z}$. have

In view of $0<r<1$ and $\left\|\chi_{O^{j}}\right\|_{\mathcal{M}_{p(\cdot), u}}^{r} \leq 2^{-j r}\|\mathcal{M} f\|_{\mathcal{M}_{p(\cdot), u}}^{r}=2^{-j r}\|f\|_{\mathcal{H}_{p(\cdot), u}}^{r}$, we

$$
\lim _{j \rightarrow-\infty} 2^{j} \int_{O^{j}}\left(\mathrm{M} \chi_{Q(x, 1)}\right)(y) d x \leq C \lim _{j \rightarrow-\infty} 2^{j-j r}\|f\|_{\mathcal{H}_{p(\cdot), u}}^{r}=0
$$

\section{References}

[1] García-Cuerva, J., and J. L. Rubio de Francia: Weighted norm inequalities and related topics. - North-Holland, 1985.

[2] Ho, K.-P.: Atomic decomposition of Hardy-Morrey spaces with variable exponents. - Ann. Acad. Sci. Fenn. Math. 40, 2015, 31-62.

Received 12 September 2016 • Accepted 16 September 2016 